\title{
Optimizing strategies in the primary Parrondo paradox
}

\author{
B. Cleuren and C. Van den Broeck \\ Limburgs Universitair Centrum, B-3590 Diepenbeek, Belgium \\ (Received 13 July 2004; published 28 December 2004)
}

\begin{abstract}
We calculate analytically the gain for the $N$-player primary Parrondo paradox and identify the optimal strategy.

DOI: 10.1103/PhysRevE.70.067104

PACS number(s): 02.50.-r, 05.40.-a
\end{abstract}

\section{INTRODUCTION}

Over the last few decades, the concepts and techniques of statistical physics have proven valuable in tackling multivariate problems in quite different fields of knowledge including traffic [1], neural networks [2], optimization [3], the immune system [4] and the stockmarket $[5,6]$. On the other hand, the constructive role of fluctuations in nonequilibrium statistical mechanics has been illustrated in various phenomena including noise-induced phase transitions, stochastic resonance, Brownian motors and resonant activation [7]. Some of these phenomena have been translated outside of their original physical context. One of them is the Parrondo paradox, stating that the alternation-random or periodic-between fair games needs no longer be a fair game [8]. In this paper, we would like to investigate a multiplayer version of the Parrondo paradox. The interest in this problem is first that one observes counterintuitive phenomena reminiscent of those found in economy and game theory, like the fact that Nash equilibria need not be Pareto optimal [9] or that greedy algorithms can lead to suboptimal solutions [10]. Second, those phenomena are now observed in models that have a physical realization (a continuous realization corresponds to a set of $N$ Brownian particles moving in a periodic asymmetric potential that can be flashed on and off at will [11]). Third and more importantly, the origin of this behavior is of a dynamic and more precisely stochastic nature, in contrast to the static equations in economy and game theory. The final reason is the general interest in exactly solvable models. Based on the experience in statistical physics, one can expect that the exact analysis of a stochastic $N$-player model is usually out of the question for a general $N$ value. Indeed, for the original multiplayer Parrondo paradox, results are only known in the limiting cases of a few ( $N$ small) or of an infinite number of players $(N \rightarrow \infty)$ [12] or by perturbation analysis around the latter state (Gaussian limit) [13]. It has even been suggested that the problem of identifying the optimal strategy in the $N$-player game belongs to the class of $N P$-complete problems [14]. Our main contribution is to show that the $N$-player version of a recently introduced simplification — the primary Parrondo paradox [15] — can be analyzed in full analytic detail for any value of $N$. Furthermore we will show that, for this particular version of the Parrondo paradox, the problem of finding the optimal strategy is polynomial rather than exponential in the number of players.

\section{PRIMARY PARRONDO PARADOX}

In the primary Parrondo paradox, a player can choose between two fair games-game $A$ and game $B$-for which the rules are (wins or losses are changes of capital with +1 and -1 , respectively) the following.

(i) Game $A$ : With probability $1 / 4$ the player wins or loses, and with probability $1 / 2$ his capital does not change.

(ii) Game $B$ : The rules for game $B$ depend on the capital of the player modulo 2 . When the capital is even, the player has a probability $4 / 9$ to win, $2 / 9$ to lose and $1 / 3$ to neither win nor lose. When his capital is odd, the player has a probability $1 / 9$ to win, $2 / 9$ to lose and $2 / 3$ to neither win nor lose.

The transition probabilities have been chosen in accordance with the fact that the games, played separately, are fair. More importantly, the transition rates are such that the resulting stochastic dynamics is superstable: the steady-state probability distribution to have an even capital-namely, $1 / 2$ for game $A$ and $1 / 3$ for game $B$-is reached after playing each of these games just once. In other words, the transition matrices associated with the Markovian dynamics for each game are idempotent operators that project on the steady distribution in one iteration. This simplification not only leads to a clear and swift discussion of the original Parrondo paradox that appears when switching between the games (see [15] for more details), but it also allows, as we proceed to show below, to give a full and detailed analytic discussion of the much more involved collective version of the game, including the identification of the optimal strategy.

\section{PRIMARY PARRONDO PARADOX WITH STRATEGY}

The collective version of the game is defined as follows. A set of $N$ players is allowed to choose, after each game, which game will be played next. Once this choice is made, all players will play the selected game, but independently of each other. The natural question arises as to which strategy should be followed to maximize the gain of the players. Since the statistics of the gains or losses only depends on the capital modulo 2 of each player and all players are equivalent, the relevant information is contained in the number of players $i \in\{0, \ldots, N\}$ with an even capital. There are $N+1$ different such configurations. Hence the state of the players after the $n$th game will be described by the probabilities $P_{i}(n)$ to find the players in these configurations $i$. We can now define the most general state-dependent strategy by in- 
troducing the probabilities $s_{i}$ to choose game $A$ (and $1-s_{i}$ to choose game $B)$ when being in state $i$. Introducing $w_{A}(j$ $\rightarrow i)$ and $w_{B}(j \rightarrow i)$ as the transition probability from configuration $j$ to $i$ when playing game $A$ or game $B$, respectively, the (discrete) time evolution of $P_{j}(n)$ is given by the following master equation:

$$
P_{i}(n+1)=\sum_{j=0}^{N}\left[s_{j} w_{A}(j \rightarrow i)+\left(1-s_{j}\right) w_{B}(j \rightarrow i)\right] P_{j}(n) .
$$

The transition probabilities $w_{A}(j \rightarrow i)$ and $w_{B}(j \rightarrow i)$ are found as follows. As mentioned before, the probability for a player to have an even capital is $1 / 2$ after playing game $A$ and $1 / 3$ after game $B$. In view of the fact that the games are played independently, the probability to have $i$ of the players in this state is given by a binomial distribution:

$$
\begin{gathered}
w_{A}(j \rightarrow i) \equiv w_{A}(i)=\left(\begin{array}{c}
N \\
i
\end{array}\right)\left(\frac{1}{2}\right)^{N}, \\
w_{B}(j \rightarrow i) \equiv w_{B}(i)=\left(\begin{array}{c}
N \\
i
\end{array}\right)\left(\frac{1}{3}\right)^{i}\left(\frac{2}{3}\right)^{N-i} .
\end{gathered}
$$

As a result of the special choice of the transition probabilities of the individual games, the transition rates for the collective game appearing in Eq. (1) factorize (such a process is called a Kangaroo process in [16]). An immediate consequence is that the steady-state solution $P_{i}^{\text {st }}=\lim _{n \rightarrow \infty} P_{i}(n)$ can be obtained explicitly:

$$
P_{i}^{\mathrm{st}}=w_{A}(i)\langle s\rangle+w_{B}(i)(1-\langle s\rangle),
$$

with $\langle s\rangle=\sum_{j=0}^{N} s_{j} P_{j}^{\text {st }}$ being the steady-state (average) probability that the next game to be played is game $A$. Note that the normalization of the $P_{i}^{\text {st }}$ follows immediately from the normalization of $w_{A}(i)$ and $w_{B}(i)$; cf. Eq. (3). The value of $\langle s\rangle$ follows from self-consistency: by multiplying Eq. (3) with $s_{i}$ and summation over $i$, one finds

$$
\langle s\rangle=\frac{s_{B}}{1-s_{A}+s_{B}},
$$

with

$$
s_{A}=\sum_{i=0}^{N} s_{i} w_{A}(i), \quad s_{B}=\sum_{i=0}^{N} s_{i} w_{B}(i),
$$

the average conditional probability to choose game $A$ when the previous game was $A$ or $B$, respectively. To complete the picture, we note that the expected gain after playing game $A$ or game $B$ when being in configuration $i$ is, respectively, given by

expected gain for game $A=0$,

$$
\text { expected gain for game } B=\frac{1}{3 N}\left(i-\frac{N}{3}\right) \text {, }
$$

where we have divided by the number of players (gain per player). We conclude that the steady-state average gain for the strategy games is given by

$$
\begin{aligned}
G\left(\left\{s_{i}\right\}\right) & =\frac{1}{3 N} \sum_{i=0}^{N}\left(i-\frac{N}{3}\right)\left(1-s_{i}\right) P_{i}^{\mathrm{st}} \\
& =\frac{1}{3 N} \sum_{i=0}^{N}\left(i-\frac{N}{3}\right)\left(1-s_{i}\right)\left[w_{A}(i)\langle s\rangle+w_{B}(i)(1-\langle s\rangle)\right] .
\end{aligned}
$$

Equation (7) is the central result of this paper: it expresses the average gain $G$ as a function of the given strategy $\left\{s_{i}\right\}$. Even though it is an exact and explicit result, the dependence

\begin{tabular}{|c|c|c|c|}
\hline$N$ & Optimal strategy & & Greedy strategy \\
\hline 1 & & $\{1,0\}$ & \\
\hline 2 & & $\{1,0,0\}$ & \\
\hline 3 & & $\{1,1,0,0\}$ & \\
\hline 4 & & $\{1,1,0,0,0\}$ & \\
\hline 5 & $\{1,1,1,0,0,0\}$ & & $\{1,1,0,0,0,0\}$ \\
\hline 6 & & $\{1,1,1,0,0,0,0\}$ & \\
\hline 7 & & $\{1,1,1,0,0,0,0,0\}$ & \\
\hline 8 & $\{1,1,1,1,0,0,0,0,0\}$ & & $\{1,1,1,0,0,0,0,0,0\}$ \\
\hline 9 & & $\{1,1,1,1,0,0,0,0,0,0\}$ & \\
\hline 10 & $\{1,1,1,1,1,0,0,0,0,0,0\}$ & & $\{1,1,1,1,0,0,0,0,0,0,0\}$ \\
\hline
\end{tabular}
on these variables is not so simple. In particular the search for the optimal strategy, leading to maximum gain, will require some further analysis.

\section{OPTIMAL STRATEGY}

The optimal strategy corresponds to the maximum of the function $G\left(\left\{s_{i}\right\}\right)$ reached in the $(N+1)$-dimensional hyper-

TABLE I. Comparison between the optimal and greedy strategy for a small number of players. For $N$ $=1,2,3,4,6,7,9$ the two strategies coincide. 


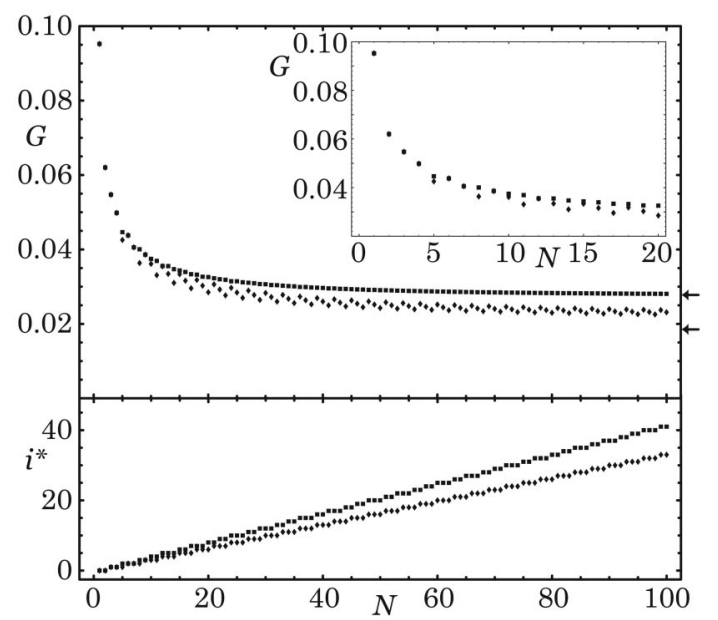

FIG. 1. Above: gain of the optimal (ם) versus greedy strategy $(\checkmark)$ as a function of the number of players $N$. The arrows indicate the limiting value of the gain as $N \rightarrow \infty$ for the optimal strategy (upper arrow) and the greedy strategy (lower arrow). Inset: blowup for a small number of players. Lower plot: threshold $i^{*}$ value for both strategies.

cube with coordinates $s_{i} \in[0,1]$. Even for a moderate number of players, searching for such a maximum numerically is an enormous and not always reliable task. In [15], we obtained the optimal strategy for $N \leqslant 5$. In Table I, we have completed the table up to $N=10$. In all cases we find that the optimal strategy lies in the corner of the hypercube, with a first set of values for the $s_{i}$, with lower index being identical to 1 , followed by the remaining set $s_{i}$ of higher index identically equal to 0 . We will now show that this is an exact and in fact general result, valid for any number of players $N$. First, we show that the value of $G$ does not decrease when, at any point in the hypercube, we replace one of the coordinates-say, $s_{k}$-by an appropriate corner value $s_{k}=0$ or $s_{k}=1$. The starting point is the evaluation of the partial derivatives of $G$ with respect to $s_{k}$ :

$$
\frac{\partial G}{\partial s_{k}}=\frac{P_{k}^{\mathrm{st}}\left[\frac{N}{2}-k+\sum_{i=0}^{N}(k-i) s_{i}\left[w_{A}(i)-w_{B}(i)\right]\right]}{3 N\left(1-s_{A}+s_{B}\right)} .
$$

Since $P_{k}^{\text {st }}>0$ [cf. Eq. (3)] and $1-s_{A}+s_{B}>0$ [see Eq. (5)], the fact whether the derivative is positive, negative, or zero depends on the expression between square brackets in Eq. (8) - namely,

$$
g_{k} \equiv \frac{N}{2}-k+\sum_{i=0}^{N}(k-i) s_{i}\left[w_{A}(i)-w_{B}(i)\right] .
$$

Furthermore, $g_{k}$ does not depend on $s_{k}$. Hence, when searching for the maximum of $G$ by varying $s_{k}$, we have only two possible situations. In the first case, $g_{k}=0$, implying $\partial G / \partial s_{k}$ $=0$ for all values of $s_{k}$. The value of $G$ does not depend on $s_{k}$ and we can choose $s_{k}$ either 0 or 1 . In the other case, the maximum must be reached on the boundary, with either $s_{k}$ $=1$ when $g_{k}>0$ or equivalently $\partial G / \partial s_{k}>0$ and $s_{k}=0$ when $g_{k}<0$ or equivalently $\partial G / \partial_{k}>0$. Since the above argument (a)

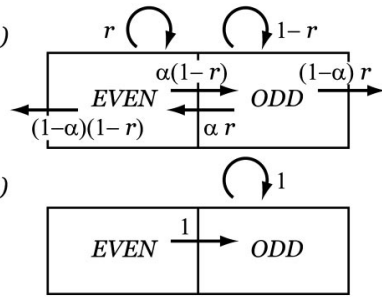

FIG. 2. (a) Schematic representation of the most general superstable and fair game with two states. The transitions are indicated by an arrow, with accompanying jump probabilities $(r \in[0,1]$ and $\alpha \in[0,1]$ ). (b) Special case with deterministic dynamics.

can be applied, starting from any point in the hypercube, and repeated iteratively until we reach one of its corners, we conclude that the maximal value of $G$ is reached in such a corner. Note that the same argument holds for locating the minimum of $G$ (by choosing at each time the corner value which yield a minimum), which is also reached in a corner of the hypercube. To proceed to the next step of the proof, we rewrite the $g_{k}$ as the following linear combination of $g_{0}$ and $g_{N}$ :

$$
g_{k}=\frac{N-k}{N} g_{0}+\frac{k}{N} g_{N}=g_{0}-\frac{k}{N}\left(g_{0}-g_{N}\right) .
$$

In the Appendix, we show that $g_{0}>0$ and $g_{N}<0$ for any strategy $\left\{s_{i}\right\}$. Hence, the $g_{k}$ are strictly decreasing as a function of $k$. The optimal strategy is thus of the form

$$
\left\{s_{i}\right\}_{\text {opt }}=\{1, \ldots, 1,1,0,0, \ldots, 0\} .
$$

We give a proof: suppose that the optimal strategy violates this ordering-say, $s_{k}=0$-followed by $s_{k+1}=1$. This would require $g_{k} \leqslant 0$ with $g_{k+1} \geqslant 0$, in contradiction with the previous finding.

The above result enormously simplifies the search for the optimal strategy. Not only does it limit our search to the corners of the $(N+1)$-dimensional space (of which their are $2^{N+1}$ in total), but we need only to check the $N+1$ corners whose coordinates have the special form Eq. (11). In other words, the time to search increases linearly rather than exponentially in $N$. This is in contrast with the situation in the original Parrondo paradox where the search for the optimal strategy is thought to be an NP-complete problem [14]. Having established this fact, we proceed to a numerical search of the optimal strategy for larger values of $N$. The results are reproduced in Fig. 1, where we plot the value $i^{*}$, defined as the configuration such that $s_{i}=1$ for $i \leqslant i^{*}$ and $s_{i}=0$ for $i$ $>i^{*}$, as a function of $N$. The dependence of $i^{*}$ on $N$ appears to have a periodic behavior: $i^{*}(N)$ presents plateaus of size 2 , $2,3,2,3$ which are repeated, at least up to the value $N$ $=100$. This suggests that $i^{*}(N) \approx(5 / 12) N$ for $N$ large, although we have not been able to prove this analytically. The optimal strategy can now be compared to the so-called greedy strategy. In this strategy game $B$ is selected whenever the total expected gain of the players is positive. Otherwise one chooses game $A$ with zero expected gain. From Eq. (6), we conclude that this strategy has the same form as the optimal strategy [cf. Eq. (11)], but with the specific threshold 
value $i^{*}=\lfloor N / 3]$. The optimal strategy always outperforms the greedy one, except for $N=1,2,3,4,6,7,9$, where they coincide (cf. Table I and Fig. 1). In the limit $N \rightarrow \infty$ the optimal strategy converges to a gain of $1 / 36 \approx 0.02777$, while the gain for the greedy strategy goes to $1 / 54$ $\approx 0.01851$ (see the arrows in Fig. $1^{1}$ ).

\section{DISCUSSION}

The above detailed analysis is presumably only possible for games that possess the property of superstability. For simplicity we have focused on a case with specific transition rates, but the above results are representative for the general case represented in Fig. 2(a). Note that an extreme example was discussed briefly in [17] [cf. Fig. 2(b)]. In game $A$ the player wins with probability 1 when the capital is even and neither wins nor loses when his capital is odd. For game $B$ the rules are the same, but with odd and even interchanged. This example exhibits the strongest possible Parrondo paradox, yet it does not produce the interesting collective features discussed here. Indeed, after having played once any one of the two games, the state (even or odd capital) of all players will be identical and will remain so forever. In this

\footnotetext{
${ }^{1}$ A mean-field calculation predicts a gain equal to $1 / 36$ for both the optimal and greedy strategies [15]. This erroneous result for the greedy strategy is understood as follows. The state of the players after playing game $B$ is described by a binomial [cf. Eq. (2)]. In the limit $N \rightarrow \infty$, this distribution converges to a $\delta$ function centered on the average $i=N / 3$. But this value exactly coincides with the threshold for the greedy strategy: i.e., play $A$ if $i \leqslant N / 3$ and play $B$ otherwise. Hence, the Gaussian fluctuations around the average value $N / 3$ cannot be ignored, and the probability to select game $A$ or $B$ is $1 / 2$ rather than 1 in this case. Note that for any other strategy, including the optimal one, with threshold strictly between $N / 3$ and $N / 2$, the problem does not occur, and the mean-field result holds.
}

sense, the multiplayer features discussed here have a genuine probabilistic nature, while the Parrondo paradox does not.

\section{ACKNOWLEDGMENT}

We acknowledge stimulating discussions with the participants of the Toledo ESF/STOCHDYN/2004 workshop.

\section{APPENDIX}

In this appendix, we show that $g_{0}>0$ and $g_{N}<0$ for any strategy $\left\{s_{0}, s_{1}, \ldots, s_{N}\right\}$. The expression of $g_{0}$ is

$$
g_{0}=\frac{N}{2}-\sum_{i=0}^{N} i s_{i}\left[w_{A}(i)-w_{B}(i)\right] .
$$

The minimal value of $g_{0}$ is obtained when the sum on the right-hand side is maximal. This maximum is obtained for the strategy $s_{i}=1$ when $w_{A}(i)-w_{B}(i)>0$ and $s_{i}=0$ otherwise. From Eq. (2) we have $w_{A}(i)-w_{B}(i)>0$ when $i>i_{c}$ $=[N \log (4 / 3) / \log (2)]$. The minimal value for $g_{0}$ is then

$$
\begin{aligned}
\left(g_{0}\right)_{\min } & =\frac{N}{2}-\sum_{i=i_{c}+1}^{N} i\left[w_{A}(i)-w_{B}(i)\right] \\
& =\sum_{i=0}^{N} i w_{A}(i)-\sum_{i=i_{C}+1}^{N} i w_{A}(i)+\sum_{i=i_{c}+1}^{N} i w_{B}(i) \\
& =\sum_{i=0}^{i_{c}} i w_{A}(i)+\sum_{i=i_{C}+1}^{N} i w_{B}(i)>0 .
\end{aligned}
$$

Here, we replaced $N / 2$ by $\sum_{i=0}^{N} i w_{A}(i)$. By a similar argument, one finds that the maximal value of $g_{N}$ is

$$
\left(g_{N}\right)_{\max }=\sum_{i=0}^{i_{c}}(i-N) w_{A}(i)+\sum_{i=i_{c}+1}^{N}(i-N) w_{B}(i)<0 .
$$

[1] D. Helbing, Rev. Mod. Phys. 73, 1067 (2001).

[2] A. Engel and C. Van den Broeck, Statistical Mechanics of Learning (Cambridge University Press, Cambridge, England, 2001).

[3] M. Mezard, G. Parisi, and M. A. Virasoro, Spin Glass Theory and Beyond (World Scientific, Singapore, 1987).

[4] S. A. Kaufman, The Origins of Order (Oxford University Press, New York, 1993).

[5] J.-P. Bouchaud and M. Potters, Theory of Financial Risks (Cambridge University Press, Cambridge, England, 2000).

[6] R. N. Mantegna and H. E. Stanley, An Introduction to Econophysics (Cambridge University Press, Cambridge, England, 2000).

[7] Unsolved Problems of Noise and Fluctuations, edited by D. Abbott and L. Kish (American Institute of Physics, Melville, New York, 2000).

[8] G. P. Harmer and D. Abbott, Nature (London) 402, 864 (1999).

[9] E. van Damme, Stability and Perfection of Nash Equilibria
(Springer-Verlag, Berlin, 1991).

[10] D. Braess, Unternehmensforschung 12, 258 (1969).

[11] F. J. Cao, L. Dinis, and J. M. R. Parrondo, Phys. Rev. Lett. 93, 040603 (2004)

[12] B. Cleuren and C. Van den Broeck, in Proceedings of SPIE, edited by Z. Gingl, J. M. Sancho, L. Schimansky-Geier, and J. Kertesz (SPIE, Bellingham, 2004), Vol. 5471.

[13] L. Dinis and J. M. R. Parrondo, Europhys. Lett. 63, 319 (2003).

[14] E. Behrends in Proceedings of SPIE, edited by Z. Gingl, J. M. Sancho, L. Schimansky-Geier, and J. Kertesz (SPIE, Bellingham, 2004), Vol. 5471.

[15] B. Cleuren and C. Van den Broeck, Europhys. Lett. 67, 151 (2004)

[16] N. G. Van Kampen, Stochastic Processes in Physics and Chemistry (North-Holland, Amsterdam, 1981).

[17] G. C. Berresford and A. M. Rockett, Int. J. Math. Math. Sci. 62, 3957 (2003). 